\title{
Glucocorticoids improve acute dizziness symptoms following acute unilateral
}

vestibulopathy.

Angel Batuecas-Caletrío ${ }^{1}$, Raquel Yañez ${ }^{1}$, Carmen Sanchez ${ }^{1}$, Pedro Blanco Pérez ${ }^{1}$, Enrique González ${ }^{1}$, Luis Alberto Guardado Sanchez ${ }^{1}$, Diego Kaski ${ }^{2 *}$

\section{Affiliations:}

1 Unidad de Otoneurología. Servicio de ORL y PCF. IBSAL. University Hospital of Salamanca, Spain

2 Department of Brain Sciences, Imperial College London, Charing Cross Hospital, London, UK

\section{* Corresponding author}

Dr Diego Kaski

Department of Brain Sciences

Imperial College London

Charing Cross Hospital

London

UK

Tel: 00442033135526

d.kaski@imperial.ac.uk

\section{The Authors declare no conflicts of interest}

Keywords

Acute unilateral vestibulopathy; Vestibular neuronitis; vertigo; glucocorticoid; steroid; symptom recovery

Word count: 1,497 (main text); 230 (abstract) 


\begin{abstract}
Background: Acute unilateral vestibulopathy (AUV) is characterized by acute vertigo, nausea, and imbalance without neurological deficits or auditory symptomatology. Here, we explore the effect of glucocorticoid treatment on the degree of canal paresis in patients with AUV, and critically, establish its relationship with dizziness symptom recovery.
\end{abstract}

Methods: We recruited consecutive patients who were retrospectively assigned to one of two groups according to whether they received glucocorticoid treatment $(n=32)$ or not $(\mathrm{n}=44)$. All patients underwent pure-tone audiometry, bithermal caloric testing, MRI brain imaging, and were asked to complete a dizziness handicap inventory on admission to hospital and just prior to hospital discharge.

Results: In the treatment group the canal paresis at discharge was significantly lower than in the control group (mean $\pm \mathrm{SD} \%: 38.04 \pm 21.57$ versus $82.79 \pm 21.51, \mathrm{p}<$ 0.001). We also observed a significant reduction in the intensity of nystagmus in patients receiving glucocorticoid treatment compared to the non-treatment group $(\mathrm{P}=$ 0.03). DHI test score was significantly lower at discharge in the treatment group (mean $\pm \mathrm{SD} \%: 23.15 \pm 12.40$ versus $64.07 \pm 12.87, \mathrm{p}<0.001)$, as was the length of hospital stay $(2.18 \pm 1.5$ days versus $3.6 \pm 1.7$ days, $\mathrm{p}=0.002)$

Conclusions: Glucocorticoid treatment leads to acute symptomatic improvement, with a reduced hospital stay and reduction in the intensity of acute nystagmus. Our findings suggest that glucocorticoids may accelerate vestibular compensation via a restoration of peripheral vestibular function, and therefore has important clinical implications for the 
treatment of AUV.

\section{INTRODUCTION}

Acute unilateral vestibulopathy (AUV) is characterized by sudden onset of rotational vertigo, nausea, and imbalance associated with intense autonomic symptoms, in the absence of neurological deficits or auditory symptoms [5]. AUV is caused by a sudden unilateral vestibular loss of function causing an asymmetry in the resting tone of the vestibular nerves that is responsible for the nystagmus and ensuing perception of vertigo. The symptoms of AUV gradually improve over time as a result of a process of vestibular compensation with eventual restitution of vestibular function in the majority of patients [1].

The aetiology of vestibular neuritis remains controversial and whilst labyrinthine ischaemia has been proposed, for the majority of patients, the natural history of the condition hints at an inflammatory cause, possibly due to reactivation of neurotropic viruses [2, 22]. One study probed the effects of corticosteroids with and without antivirals and concluded that methylprednisolone, but not valacyclovir, improves the recovery of vestibular function in these patients [21]. Other studies have confirmed the effects of glucocorticoids on peripheral vestibular function following acute AUV [18, 20].

Surprisingly however, only one study has indirectly assessed the effects of glucocorticoids on dizziness symptoms in AUV, finding no long-term benefit [20]. The focus has instead been on canal paresis recovery, a surrogate marker of the vestibuloocular reflex. Despite asymmetrical VOR time constants following an acute vestibulopathy, recent evidence has shown a considerable dissociation between vestibulo-ocular and vestibulo-perceptual thresholds (i.e. the perception of self-motion) [7]. This poses the question of whether restoring the vestibular tone imbalance acutely and thus reducing nystagmus - through a reduction in inflammation would have any effect on dizziness symptoms (i.e. whether central compensatory mechanisms actuate to 
reduce self-motion perception (and thus dizziness) too quickly for glucocorticoids to be effective).

The primary aim of this study was therefore to explore the acute effect of glucocorticoid treatment on dizziness symptom recovery and length of hospital stay.

\section{MATERIALS AND METHODS}

We recruited consecutive patients with a clinical diagnosis of AUV presenting to the Department of Oto-Rhino-Laryngology, Head \& Neck Surgery at Salamanca University Hospital between March 2005 and April 2012. All patients were assessed by two experienced neuro-otologists to ensure that diagnostic criteria were rigorous and consistent throughout the recruitment period. Diagnostic criteria for AUV included a history of sudden onset of dizziness without auditory or neurological symptoms, contralesional spontaneous unidirectional horizontal-rotational nystagmus, increasing in severity when visual fixation is abolished, ipsilesional abnormal head thrust test, and gait unsteadiness. Our centre has routinely offered glucocorticoid treatment to all patients with AUV since September 2007.

All patients underwent pure-tone audiometry in the first or second day after the onset of symptoms, and a Fitzgerald and Hallpike bithermal caloric test on the fourth or fifth day, using video-oculography (Ulmer VNG, v. 1.4, SYNAPSIS, Marseille, France) that enables an objective measure of peripheral vestibular function and symmetry [9]. The maximum slow phase velocity (SPVmax) of nystagmus was calculated following each irrigation using an automated analysis incorporated into the system. The total caloric response from each ear (TotE) was calculated, and caloric weakness (CW) and directional preponderance (DP) were determined according to Jongkees' formula. Note that caloric testing was not performed prior to starting glucocorticoid therapy given that a thorough neuro-otological examination is felt to be sufficiently sensitive to correctly identify an acute unilateral vestibulopathy [24], and to avoid delays in initiating treatment. Additionally, in accordance with local clinical guidelines for patients presenting with an acute vestibulopathy, all patients underwent magnetic resonance imaging (MRI) of the brain on the fourth or fifth day following symptom onset. 
Video head impulse test and vestibular evoked myogenic potential data was not available for the majority of the patients tested, given the relatively recent introduction of these techniques into routine clinical practice, and are therefore not reported here.

\section{The Dizziness Handicap Inventory}

Dizziness Handicap Inventory (DHI) was also completed by the patient at the time of hospital discharge (mean duration to completion was 4.2 days). The DHI is a qualitative questionnaire used to quantify the self-perceived level of handicap associated with the symptom of dizziness in everyday life. It is a widely used, validated, and standardized test for patients with dizziness and unsteadiness [14, 23].

Patients were retrospectively assigned to one of two groups according to whether they received glucocorticoid treatment or not, as part of a cohort study. All patients underwent daily review by a Consultant otolaryngologist during the in-patient stay. All patients were additionally treated with intravenous diazepam 5mg twice daily for the first and second day only to treat potential autonomic symptomatology. Nausea and vomiting were treated with intravenous metoclopramide 10mg every 8 hours during the first day. Patients were encouraged to mobilise as soon as possible, and were instructed to follow self-guided validated Cawthorne-Cooksey vestibular rehabilitation exercises $[6]$.

\section{Glucocorticoid treatment group}

Patients in this group were consecutively recruited between September 2007 and April 2012. Patients were treated with intravenous methylprednisolone $1 \mathrm{mg} / \mathrm{kg}$ for 5 days. The dosage was subsequently halved for another five days and then discontinued. The treatment protocol was based on local guidelines for the treatment of AUV established in 2007 and is similar to that used in Shupak et al. [20] and Karlberg \& Magnusson [15].

\section{Control group}

Patients in the no-glucocorticoid group were consecutively recruited between March 2005 and September 2007. In this group, patients were treated symptomatically with the same drugs and dosage as the treatment group patients but did not receive glucocorticoid. 


\section{Statistical analysis}

Student's $t$ test for unpaired observations was used to compare the data of the glucocorticoid treatment group with the control groups, following confirmation that the data met assumptions of equal variances (verified with Levene's test for equality of variances) and the data was normally distributed (Kolgomorov-Smirnov test). The analysis was performed using SPSS Statistics version 19. A $p<0.05$ was considered to be statistically significant.

\section{RESULTS}

\section{Clinical data}

Demographic and clinical data are presented in Table 1. The glucocorticoid treatment group comprised 32 patients (17 men and 15 women) and the control group 44 patients (23 men and 21 women). Age was not statistically different between groups (mean \pm SD age: $51.74 \pm 15.32$ versus $57.51 \pm 18.35$ years, $p=0.18$ Student's $t$ test for unpaired observations). No major adverse effects of the glucocorticoid treatment group were observed but glycemic control was needed in five patients.

\begin{tabular}{|cccc|}
\hline & $\begin{array}{c}\text { Glucocorticoids treatment } \\
\text { group } \\
(\mathbf{n = 3 2})\end{array}$ & $\begin{array}{c}\text { Control group } \\
(\mathbf{n}=\mathbf{4 4})\end{array}$ & P value \\
\hline Gender (M/F) & $17 / 15$ & $23 / 21$ & $\mathrm{NS}$ \\
\hline Age (yrs) & $51.74 \pm 15.32$ & $57.51 \pm 18.35$ & $\mathrm{NS}$ \\
\hline $\begin{array}{c}\text { Lesion side } \\
\text { (R/L) }\end{array}$ & $19 / 13$ & $26 / 18$ & $\mathrm{NS}$ \\
\hline $\begin{array}{c}\text { Symptom onset } \\
\text { to admission } \\
\text { (hrs) }\end{array}$ & $20.45 \pm 7.16$ & $17.36 \pm 5.87$ & $\mathrm{NS}$ \\
\hline $\begin{array}{c}\text { Nystagmus type } \\
\text { on admission } \\
\text { (grade I; grade } \\
\text { II; grade III) }\end{array}$ & $0 ; 5 ; 27$ & $0 ; 8 ; 36$ & $\mathrm{NS}$ \\
\hline $\begin{array}{c}\text { Nystagmus type } \\
\text { at discharge } \\
\text { (grade I; grade } \\
\text { II; grade III) }\end{array}$ & $20 ; 8 ; 4$ & $21 ; 15 ; 8$ & $\mathrm{P}=0.03$ \\
\hline $\begin{array}{c}\text { Canal paresis at } \\
\text { discharge }\end{array}$ & $38.04 \pm 21.57$ & $82.79 \pm 21.51$ & $\mathrm{P}<0.001$ \\
\hline
\end{tabular}




\begin{tabular}{|cccc|}
\hline $\begin{array}{c}\text { Length of } \\
\text { hospital stay } \\
\text { (days) }\end{array}$ & $2.8 \pm 1.5$ & $3.6 \pm 1.7$ & $\mathrm{P}=0.002$ \\
\hline Discharge DHI & $23.15 \pm 12.40$ & $64.07 \pm 12.87$ & $\mathrm{P}<0.001$ \\
\hline
\end{tabular}

Table 1: Demographic and clinical data across both groups. NS=not significant $(\mathrm{p}>0.05)$.

Magnetic resonance imaging of the brain was normal in all patients in the treatment group but four patients were excluded from the study on the basis of abnormal findings on magnetic resonance imaging of the brain ( 2 patients with infarcts in the territories of the posterior inferior cerebellar artery and 2 patients with cerebellopontine angle lesions).

In-patient hospital stay differed significantly between groups $(2.8 \pm 1.5$ days for the treatment group and 3.6 \pm 1.7 days for the control group; $\mathrm{P}=0.002$ ).

\section{Canal paresis}

Group data showed that canal paresis was significantly lower in the treatment group compared to the control group (mean \pm SD \%: $38.04 \pm 21.57$ versus $82.79 \pm 21.51, p<$ 0.001 Student's $t$ test for unpaired observations).

\section{Oculomotor function}

At hospital admission 27 patients from the treatment group had grade III nystagmus, and 5 had grade II, compared to 36 with grade III, and 8 with grade II nystagmus in the control group ( $\mathrm{p}=0.07$; ANOVA).

At hospital discharge, 20 patients from the treatment group had grade I nystagmus, 8 grade II nystagmus, and 4 grade III nystagmus, whereas in the control group, 21 had grade I nystagmus, 15 grade II nystagmus, and 8 grade III nystagmus $(\mathrm{P}=0.03$; ANOVA).

\section{Dizziness Handicap Inventory}

DHI test score at discharge was significantly lower in the treatment group (mean \pm SD $\%: 23.15 \pm 12.40$ (physical $(\mathrm{P})=8.05$; functional $(\mathrm{F})=7.47$; emotional $(\mathrm{E})=8.13$ ) versus 
$64.07 \pm 12.87(\mathrm{P}=20.31 ; \mathrm{F}=19.56 ; \mathrm{E}=24.06), p<0.001$ Student's $t$ test for unpaired observations).

\section{DISCUSSION}

This study confirms prior reports showing peripheral vestibular function improvement in patients with AUV receiving glucocorticoids on admission [3, 20, 21]. Additionally, we have shown that the improvement in canal paresis with glucocorticoid treatment correlates with a reduction in the degree of nystagmus. Critically, however, we show here that acute glucocorticoid treatment reduces symptom load and hospitalization in patients with AUV.

Previous studies hinted that glucocorticoids may not be clinically beneficial in AUV [10], despite apparent improvement in vestibular function $[3,15,17,20]$. Indeed, both a systematic review [13] and later a Cochrane review of four trials found no significant difference between corticosteroid and placebo in the symptomatic recovery of vestibular function [11], but only one study had assessed symptom recovery [20], and this was not a primary study outcome. The short follow-up period in our study does not make the results with those of Shupak et al. [20] directly comparable, but one possibility is that the reduction in acute dizziness symptoms was related to the psychotropic effects of glucocorticoids [8] in our treatment group. Against this hypothesis is that euphoric sequelae of steroids in some patients (better DHI scores) would perhaps be counterbalanced by depressive and anxiety-related side-effects [8] in other patients (worse DHI scores). Moreover, that improvements were seen with glucocorticoids across all functional, physical, and emotional DHI subscores further suggests that the effects of treatment are not limited to emotional aspects only (as one might expect for purely psychotropic steroid effects). Whether or not psychotropic effects of glucocorticoids have influenced the lower dizziness scores in these patients, the consequent reduction in hospital stay in the treatment group is a consistent [15] and important clinical finding where healthcare resources are financially constrained. Furthermore, patients with acute symptomatic improvement may be less likely to develop secondary non-vertiginous psychological symptoms in the long term, thus reducing chronic disease burden in this patient population. Further work will be needed to explore this concept. 
An alternative, non-mutually exclusive hypothesis to explain the lower DHI scores in the treatment group is that glucocorticoid treatment may have an acute physiological effect on the vestibular nerve. Treatment with glucocorticoids in animal models of unilateral vestibular failure accelerates vestibular compensation - a central phenomenon triggered by a disturbance in peripheral firing rates of the vestibular nerve [19]. Reducing inflammation acutely with glucocorticoids may thus restore ipsilesional vestibular tone and thus reduce the degree of asymmetry, facilitating compensatory processes. Indeed, the improvement in the degree of nystagmus at hospital discharge in the glucocorticoid arm suggests that the glucocorticoids may be preferentially acting at the peripheral level, rather than centrally.

Our study has some limitations. Firstly, whilst short-term symptomatic improvement in patients receiving glucocorticoids, in conjunction with reduced hospital stay, may be sufficient clinical indication to treat AUV with glucocorticoids acutely, an appreciation of long-term symptomatic outcomes remains an important unanswered question. The clinical use of glucocorticoids to reduce acute symptoms without altering the natural course of the condition is however an established practice in neurology, for example in multiple sclerosis [12]. Secondly, patients were retrospectively assigned to the treatment group based on current and previous clinical practice. Lastly, we have used a single measure of symptomatic recovery in patients. Nevertheless, whilst other dizziness questionnaires explore symptom severity and psychological measures of anxiety and depression in more depth, the DHI is a simple, clinically-applicable test that is sensitive to change with interventions [4] and correlates well with objective measures of balance [16].

In conclusion, our data suggest that glucocorticoids may accelerate vestibular compensation perhaps via a restoration of peripheral vestibular function, given the observed reduced nystagmus intensity in the treatment group. This finding has important clinical implications for the treatment of AUV, although further studies will need to assess whether acute administration of glucocorticoid treatment alters long-term outcome in these patients, and directly explore the underlying mechanism of human vestibular compensation in relation to glucocorticoid administration.

\section{REFERENCES}


1. Arbusow V, Schulz P, Strupp M, Dieterich M, von Reinhardstoettner A, Rauch E, Brandt T (1999) Distribution of herpes simplex virus type 1 in human geniculate and vestibular ganglia: implications for vestibular neuritis. Ann Neurol 46:416-419

2. Arbusow V, Theil D, Strupp M, Mascolo A, Brandt T (2001) HSV-1 not only in human vestibular ganglia but also in the vestibular labyrinth. Audiol Neurootol 6:259-262

3. Ariyasu L, Byl FM, Sprague MS, Adour KK (1990) The beneficial effect of methylprednisolone in acute vestibular vertigo. Arch Otolaryngol Head Neck Surg 116:700-703

4. Badke MB, Miedaner JA, Shea TA, Grove CR, Pyle GM (2005) Effects of vestibular and balance rehabilitation on sensory organization and dizziness handicap. Ann Otol Rhinol Laryngol 114:48-54

5. Baloh RW (2003) Clinical practice. Vestibular neuritis. The New England journal of medicine 348:1027-1032

6. Corna S, Nardone A, Prestinari A, Galante M, Grasso M, Schieppati M (2003) Comparison of Cawthorne-Cooksey exercises and sinusoidal support surface translations to improve balance in patients with unilateral vestibular deficit. Archives of physical medicine and rehabilitation 84:1173-1184

7. Cousins S, Kaski D, Cutfield N, Seemungal B, Golding JF, Gresty M, Glasauer S, Bronstein AM (2013) Vestibular perception following acute unilateral vestibular lesions. PLoS One 8:e61862

8. Dubovsky AN, Arvikar S, Stern TA, Axelrod L (2012) The neuropsychiatric complications of glucocorticoid use: steroid psychosis revisited. Psychosomatics 53:103-115

9. Eggers SD, Zee DS (2003) Evaluating the dizzy patient: bedside examination and laboratory assessment of the vestibular system. Seminars in neurology 23:47-58

10. Fishman JM (2011) Corticosteroids effective in idiopathic facial nerve palsy (Bell's palsy) but not necessarily in idiopathic acute vestibular dysfunction (vestibular neuritis). Laryngoscope 121:2494-2495

11. Fishman JM, Burgess C, Waddell A (2011) Corticosteroids for the treatment of idiopathic acute vestibular dysfunction (vestibular neuritis). Cochrane Database Syst Rev:CD008607

12. Goodin DS (2014) Glucocorticoid treatment of multiple sclerosis. Handbook of clinical neurology 122:455-464

13. Goudakos JK, Markou KD, Franco-Vidal V, Vital V, Tsaligopoulos M, Darrouzet V (2010) Corticosteroids in the treatment of vestibular neuritis: a systematic review and meta-analysis. Otol Neurotol 31:183-189

14. Jacobson GP, Newman CW (1990) The development of the Dizziness Handicap Inventory. Arch Otolaryngol Head Neck Surg 116:424-427

15. Karlberg ML, Magnusson M (2011) Treatment of acute vestibular neuronitis with glucocorticoids. Otol Neurotol 32:1140-1143

16. Kaufman KR, Brey RH, Chou LS, Rabatin A, Brown AW, Basford JR (2006) Comparison of subjective and objective measurements of balance disorders following traumatic brain injury. Medical engineering \& physics 28:234-239

17. Kitahara T, Kondoh K, Morihana T, Okumura S, Horii A, Takeda N, Kubo T (2003) Steroid effects on vestibular compensation in human. Neurological research 25:287-291 
18. Kitahara T, Okumura S, Takeda N, Nishiike S, Uno A, Fukushima M, Kubo T (2001) [Effects of steroid therapy on long-term canal prognosis and activity in the daily life of vestibular neuronitis patients]. Nihon Jibiinkoka Gakkai kaiho 104:1059-1064

19. Olabi B, Bergquist F, Dutia MB (2009) Rebalancing the commissural system: mechanisms of vestibular compensation. J Vestib Res 19:201-207

20. Shupak A, Issa A, Golz A, Margalit K, Braverman I (2008) Prednisone treatment for vestibular neuritis. Otol Neurotol 29:368-374

21. Strupp M, Zingler VC, Arbusow V, Niklas D, Maag KP, Dieterich M, Bense S, Theil D, Jahn K, Brandt T (2004) Methylprednisolone, valacyclovir, or the combination for vestibular neuritis. The New England journal of medicine 351:354-361

22. Theil D, Arbusow V, Derfuss T, Strupp M, Pfeiffer M, Mascolo A, Brandt T (2001) Prevalence of HSV-1 LAT in human trigeminal, geniculate, and vestibular ganglia and its implication for cranial nerve syndromes. Brain Pathol 11:408-413

23. Treleaven J (2006) Dizziness Handicap Inventory (DHI). The Australian journal of physiotherapy 52:67

24. Walker MF, Zee DS (2000) Bedside vestibular examination. Otolaryngol Clin North Am 33:495-506

\section{Figure legends}

Figure 1: Box plots showing $\boldsymbol{A}$ percentage vestibular canal paresis and $\boldsymbol{B}$ dizziness handicap inventory (DHI) test scores at discharge from hospital in the glucocorticoid and no-glucocorticoid patient groups. The lines inside the boxes show the median, the extent of the box shows the $25^{\text {th }}$ and $75^{\text {th }}$ percentiles. Whiskers extend to the furthest observation within $1.5 \mathrm{x}$ inter-quartile range of the $25^{\text {th }} / 75^{\text {th }}$ percentile. 